\title{
Combining interventions to reduce the spread of viral misinformation
}

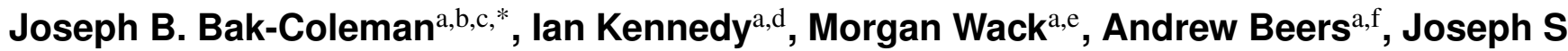 \\ Schafer ${ }^{\mathrm{a}, \mathrm{f}}$, Emma S. Spiro ${ }^{\mathrm{a}, \mathrm{c}, \mathrm{d}}$, Kate Starbird ${ }^{\mathrm{a}, \mathrm{g}}$, and Jevin D. West ${ }^{\mathrm{a}, \mathrm{c}}$
}

${ }^{a}$ Center for an Informed Public, University of Washington, Seattle, WA 98195

beScience Institute, University of Washington, Seattle, WA 98195

'The Information School, University of Washington, Seattle, WA 98195

${ }^{\mathrm{d} D e p a r t m e n t}$ of Sociology, University of Washington, Seattle, WA 98195

eDepartment of Political Science, University of Washington, Seattle, WA 98195

fPaul G. Allen School of Computer Science and Engineering, University of Washington, Seattle, WA 98195

'Human Centered Design and Engineering, University of Washington, Seattle, WA 98195

*Corresponding Author: joebak@uw.edu

\begin{abstract}
Misinformation online poses a range of threats, from subverting democratic processes to undermining public health measures. Proposed solutions range from encouraging more selective sharing by individuals, to the removal of false content and accounts that create or promote it. Here we provide a framework to evaluate interventions aimed at reducing viral misinformation online both in isolation and when used in combination. We begin by deriving a generative model of viral misinformation spread, inspired by research on infectious disease. Applying this model to a large corpus of misinformation events that occurred during the 2020 US election, we reveal that commonly proposed interventions are unlikely to be effective in isolation. However, our framework demonstrates that a combined approach can achieve a substantial $(\approx 50 \%)$ reduction in the prevalence of misinformation. Our results highlight a practical path forward as misinformation online continues to threaten vaccination efforts, equity, and democratic processes around the globe.
\end{abstract}

\section{Introduction}

Misinformation-i.e., false information- has become a pervasive feature of online discourse, resulting in increased belief in conspiracy theories, rejection of recommended public health interventions, and even genocide ${ }^{1-3}$. Academics and those working in industry have proposed a host of potential solutions, ranging from techniques for detecting and removing misinformation to empowering users to be more discerning in their sharing habits ${ }^{4-6}$. Despite an abundance of proposed interventions, online misinformation remains a global prob$\mathrm{lem}^{7-9}$. For instance, the 2020 US Presidential election and subsequent insurrection at the Capitol building highlighted how pervasive online misinformation online can lead to realworld harm.

That real-world violence occurred as a result of a broader narrative that questioned the election's legitimacy, which arose from a series of more specific claims. Most claims were characterized by a brief period (i.e., hours, days) of rapid growth in discussion and sharing ${ }^{2}$. During these events, engagement (i.e., all discussion and sharing) exhibits viral, disease-like dynamics — self-replicating, endogenous growth stemming from a limited number of initial sources ${ }^{10,11}$. Some of these incidents quickly died out, while others had multiple waves, spread to other platforms, and often became consolidated into broader narratives.

Early response provides a source of promise for successful intervention, as disrupting viral spread may have cascading effects on narrative consolidation and future engagement. Un- fortunately, the rapid growth inherent to viral misinformation makes it challenging to assess and respond to in a timely manner. Effective intervention by platforms and policymakers requires a temporally-aware framework for quantitative comparison of proposed interventions.

Lacking this, it is unclear whether existing strategies are sufficient to produce meaningful results. Crude approaches like outright removal and banning of either content or accounts will certainly work if applied in excess, yet come with costs to freedom of expression and force private entities to be arbiters of truth. For judicious use, questions arise about how soon and how much removal is necessary for a meaningful effect. Similarly, interventions that rely on empowering individuals to consume and share more discerningly have shown promise in experimental contexts. Still, it remains unclear what impact they will have at scale ${ }^{5}$.

Beyond comparison, we lack an understanding of whenand indeed whether-multiple interventions can act synergistically to reduce the spread of misinformation. Unfortunately, experiments do not adequately address these questions as their efficacy at scale cannot be directly inferred. For example, the consequences of variation in follower counts, which span eight orders of magnitude, would be difficult to capture in the lab. Moreover, the unique behavior of highly influential repeat spreaders (e.g., coordination, early amplification) will certainly impact dynamics and thus efficacy ${ }^{2}$. Further, the time scales at which viral misinformation events occur online (i.e., hours, days, weeks) pose challenges to extrapolation 
from comparatively brief experiments.

Platforms like Twitter can and do run experiments at scale, yet the data and methods are not generally made available for open research. Moreover, private companies conduct these experiments in the absence of scientific discourse or ethical oversight. While we can observe some changes in response to platform policies, disentangling the effect of the policy from unseen algorithmic alterations, interface modifications, or behavioral change is nearly impossible ${ }^{12}$.

Despite these challenges, insight is urgently required as poorly implemented policies or inaction could exacerbate misinformation and cost lives ${ }^{13}$. Toward this goal, we derive and parameterize a generative model of misinformation engagement (i.e., total discussion and sharing of posts related to false information) using a large corpus of tweets collected during the 2020 election in the $\mathrm{US}^{2}$. This approach captures the dynamics of viral misinformation at scale and across time in a real-world context. We rely on this model to examine the efficacy of misinformation interventions both in isolation and when deployed in combination. Finally, we examine how the spread of misinformation during viral periods impacts subsequent engagement.
A

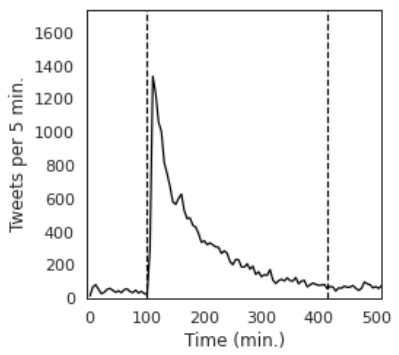

C

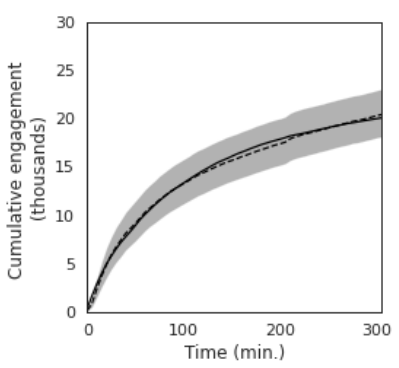

B

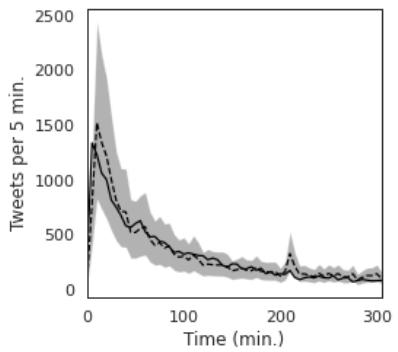

D

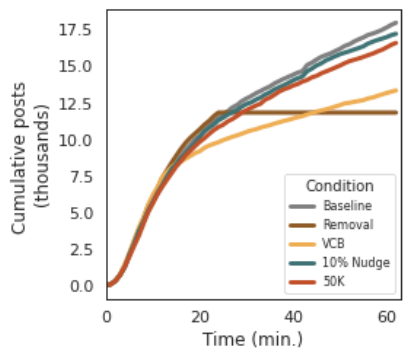

Figure 1. Overview of our model and analysis A) An event segmented from a larger incident (dashed lines). B) timeseries for a single event. Dashed-line: expected value, shaded region: $89 \%$ credible interval (CI). C) Cumulative engagement as a measure of total misinformation, lines and shading are as in B. D) Model-simulated platform interventions for a single event. Lines indicate median cumulative engagement over 100 simulations. Grey: baseline, blue: $10 \%$ "nudge", orange: banning, yellow: virality circuit breaker, brown: outright removal of content

\section{Results}

\section{Data and Model Overview}

Our analysis relies on a dataset of Twitter posts collected during the 2020 US election. This dataset was extracted from a broader collection of 1.04B election-related tweets collected between September 1, 2020, and December 15, 2020. To construct our dataset, we first identified 430 incidentsdistinct stories that included false, exaggerated, or otherwise misleading claims or narratives. Search terms were devised for each incident, extracting $23 \mathrm{M}$ tweets generated by $10.8 \mathrm{M}$ accounts from the broader collection.

From each incidents' time series, we extracted events, defined as periods in which a story exhibited rapid growth and decay (See Methods, Fig. 1A). This process identified 544 potentially viral events, including 14.6M Twitter posts (Tweets, Retweets, Replies and Quote Tweets).The number of viral events (544) is higher than the number of incidents (430) because the incidents could have more than one viral event. We then derived a generative model of viral information spread. We adapted a model of super-spreading from the infectious disease literature to characterize the spread of misinformation and efficacy of interventions. Our model treats virality as temporally varying, increasing proportionally to the out-degree (i.e., the number of followers) of the accounts that post about a topic. We also assume that virality decays over time as the network saturates and new topics arise. Details can be found in the Methods.

Using Bayesian methods, we estimated model parameters for each event (Fig. 1B-C). As our model is not expected to fit all topics discussed during the election, we developed inclusion criteria to ensure our model was appropriate for a given event and that the derived parameters could reproduce the observed engagement (see Methods). This led to our final dataset of 9.7M tweets from 457 events. We then simulated total engagement by seeding the model with the estimated parameters, posts in the initial 5 minute timestep, and the empirical distribution of follower counts for each five-minute interval. Our simulated engagement strongly corresponded to observed engagement for events spanning several orders of magnitude in post volume (Fig S2). For the results presented throughout, we modified our model in various ways and evaluated the total simulated engagement across all included events (Fig. 1D, See Methods).

\section{Fact-Checking and time-lagged approaches}

We begin by considering the impact on user engagement (i.e., total posts: retweets, tweets, quote-tweets, and replies) of approaches in which a platform applies policies that target a specific instance of misleading or false information-in this case, individual posts. Among the more commonly employed strategies during the 2020 US election was identifying specific misinformation and taking action, ranging from applying a label to outright removal ${ }^{2}$. These approaches share a common feature of requiring time before action is taken. Time is necessary not only to identify misinformation but also to decide 


\section{A}

C

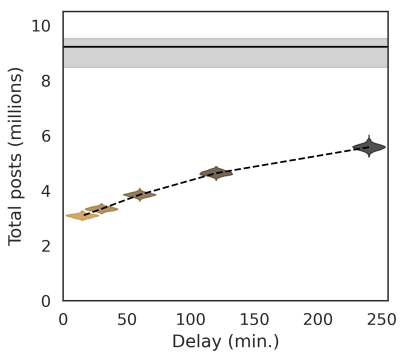

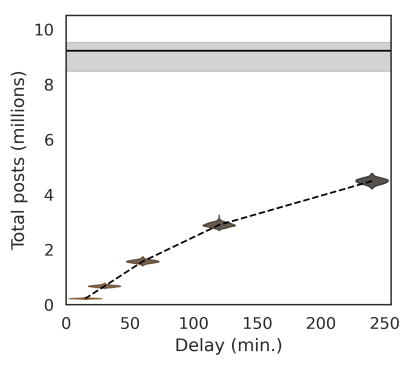

B

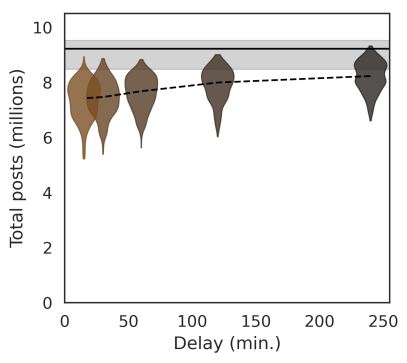

D

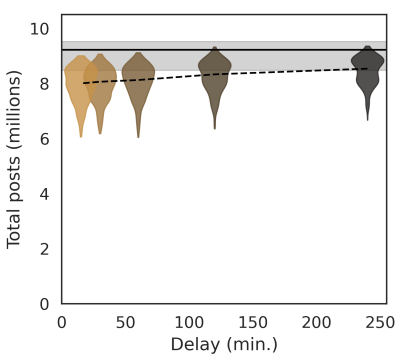

Figure 2. A) The impact of outright removal on of all misinformation-related posts following a delay specified in minutes. B) As in A if only $20 \%$ of events are removed. C) The impact of applying a virality circuit breaker that reduces virality by $10 \%$ to all misinformation events after a specified period of time. D) As in C, if the VCB is applied to only $20 \%$ of events. Horizontal gray bars in each plot represent baseline conditions, with the line indicate the mean and shaded area highlighting the $89 \%$ credible interval. Violins indicate the simulated distribution of total posts across all events.
A more plausible approach could involve "virality circuit breakers" (VCBs) which seek to reduce the spread of a trending misinformation topic without explicitly removing content, for example, by suspending algorithmic amplification ${ }^{14}$. This approach allows platforms to consider ethical ramifications while minimizing the public relations challenges accompanying direct forms of action. This could aid in lowering the threshold for fact-checking, fact-checking and therefore enable quicker response times. We simulate the impact of virality circuit breakers by proportionately reducing the latent virality parameter in our model after a fixed time interval.

Through simulations, we reveal how virality circuit breakers can have similar efficacy to outright removal even if the amount by which virality is reduced is small (Fig. 2C, Table S3). For instance, a $10 \%$ reduction in virality implemented four hours after the start of an event can reduce the spread of misinformation by nearly $39.3 \%$ (89\% C.I. [34.4, 42.2]). As with outright removal, however, the efficacy is primarily limited by the proportion of events for which the platforms take action (Fig 2D, Table S3)

\section{Nudges and reduced reach}

A drawback of fact-checking-based approaches is that they are most applicable to transparently false or readily falsifiable claims $^{2}$. Many instances of misinformation involve claims that are either partly true or require non-trivial time to debunk. Claims that there are statistical irregularities in reported vote tallies, for example, require a statistician gathering and analyzing the data and determining merit. Depending on the implementation, time-lagged responses may require users to be receptive to the intervention and do not devise workarounds.

These challenges motivate approaches that leverage individual discretion to reduce to spread of misinformation ${ }^{6}$. For instance, encouraging users to consider accuracy has been shown to improve discernment of false information sharing by $10-20 \%$. This can be implemented by warning users when they encounter potentially false or misleading information, but this still requires the labeling of that content as false as it does for fact-checking.

A central question is whether a modest reduction in individual sharing behavior can lead to a more dramatic change in overall rates of misinformation. Agent-based models support this notion across a range of network topologies ${ }^{5}$. From the perspective of our model, nudge-based approaches can be simulated by maintaining the parameters from the initial model fit while proportionally reducing the following of every user that discusses an incident.

Using our model to simulate nudges, we find that they can indeed reduce the prevalence of misinformation (Fig 3A, Table S5). Nudges that reduce sharing by 5, 10, 20, and $40 \%$ result in a $7.9,16.1,28.8$, and $47.7 \%$ reduction in cumulative engagement, respectively (Table S5). The median effect tends to be larger than the nudge suggesting a degree of feedback whereby the individual effect of a nudge is compounded in the misinformation dynamics.

\section{Account Banning}

In our dataset, several accounts shared or amplified misinformation across multiple incidents ${ }^{2}$. Moreover, some of these repeat offenders had outsized audiences compared to the average Twitter user-ranging from hundreds of thousands to millions of followers. While removal of repeat offenders during the election was rare, several were removed following the 
violent insurrection at the U.S. Capitol on January 6, 2021. A question remains whether removing these accounts or accountfocused policies, in general, would have a meaningful impact on misinformation. While large followings often confer engagement, it remains possible that there is sufficient sharing from smaller accounts to ensure the spread of misinformation even in the absence of the larger removed accounts ${ }^{15}$.

One challenge in modeling account removal is that there likely exist non-trivial relationships between account size, the propensity to share misinformation, and the timing at which certain accounts amplify narratives. A large account that regularly shares misinformation in the first five minutes will have an outsized effect compared to a smaller account that occasionally shares misinformation hours later. To account for this, our model samples from the empirical follower-count distribution in a given timestep. Further, as the identities of individuals are known, we can remove specific accounts and simulate total engagement (See Methods). In other words, our simulations are conditioned on unseen patterns of, and variation in, individual behavior without explicitly quantifying the differences in individual behavior. Through this, our model and simulations exhibit robustness to considerable unmeasured real-world complexity.

We begin by considering the consequences of account removals $(N=1504)$ in early 2021 . We seek to answer whether previously implemented account removal is sufficient to curb misinformation going forward. Our simulations reveal that the removal of these accounts from our dataset reduces total engagement with misinformation by $18 \%$, (89\% C.I. [10.2, 22.7], Fig 3C). This is comparable in efficacy to a $10 \%$ reduction in sharing of misinformation (i.e., a nudge) impacting all accounts in the absence of removal.

We next consider a "3 Strikes" rule in which accounts are removed from the platform after they are detected in three separate incidents of misinformation (i.e., topics, regardless of the number of posts for a given topic). For these simulations, any interaction or amplification of misinformation (i.e., tweets, retweets, quote tweets) would be counted as a strike. A policy focused solely on original content could be gamed by using large accounts to amplify disposable smaller accounts. This type of policy would avoid banning accounts that were swept up by a given piece of misinformation and repeatedly tweeted while focusing on those that spread misinformation more broadly. Applied solely to verified accounts, we observe an $11.3 \%$ drop in cumulative engagement (89\% C.I. $[3.2,18.7])$, which likewise is similar in efficacy to a small nudge rolled out across the board (Table S6, Fig. 3C). If, instead of verification, the policy is applied based on the number of followers an account has, pronounced effects are only observed when the threshold is quite low $(\approx 10 \mathrm{~K}$ followers) requiring large numbers of accounts to be removed (Fig. 3B,D, Table S7).
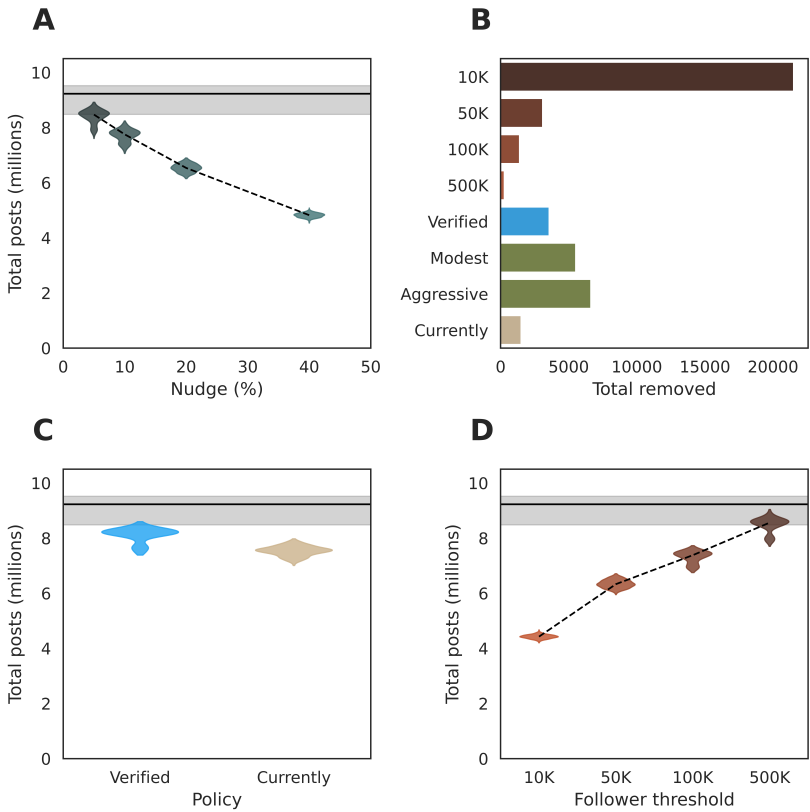

Figure 3. A) The effect of nudges that inoculate a percentage of the population against spreading misinformation. Shown is the cumulative total engagement across all events. B) Number of accounts that are either currently removed, or would have been removed under a three-strikes policy $\mathbf{C}$ ) The effect of accounts removal for either those that are currently banned (orange) or those banned following a three strikes rule applied solely to verified accounts (blue). D) As in A and C, yet showing the impact of enacting three-strikes policies with varying thresholds. Horizontal gray bars in A, B and C represent baseline conditions, with the line indicate the mean and shaded area highlighting the $89 \%$ credible interval. Violins indicate the simulated distribution of total posts across all events.

\section{Combined Approaches}

All of the approaches above exhibit some efficacy in reducing engagement with viral misinformation. Unfortunately, each strategy tends to become maximally effective in impractical regions of parameter space. Outright removal of misinformation is particularly effective, yet it is difficult to imagine that more than a small fraction of misinformation can be easily removed. Virality circuit breakers face similar challenges, albeit to a lesser extent. For nudges that minimally impact user experience yet improve individual discretion, effects far beyond $\approx 20 \%$ are unlikely without a significant breakthrough in information literacy or social psychology ${ }^{5}$. In the case of banning specific accounts, low follower thresholds increase the number of accounts removed, and thus costs and challenges, super-linearly.

We, therefore, consider a combined approach relying on only modest implementations of each of the strategies studied above. Specifically, viral circuit breakers are employed for $5 \%$ of content, reducing virality by $10 \%$, and enacted after 

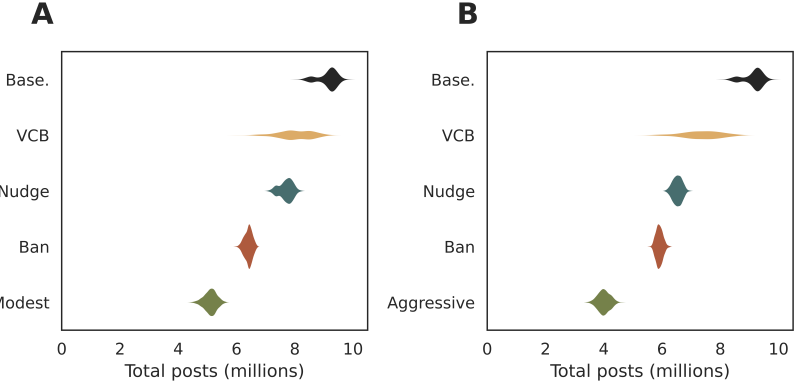

C
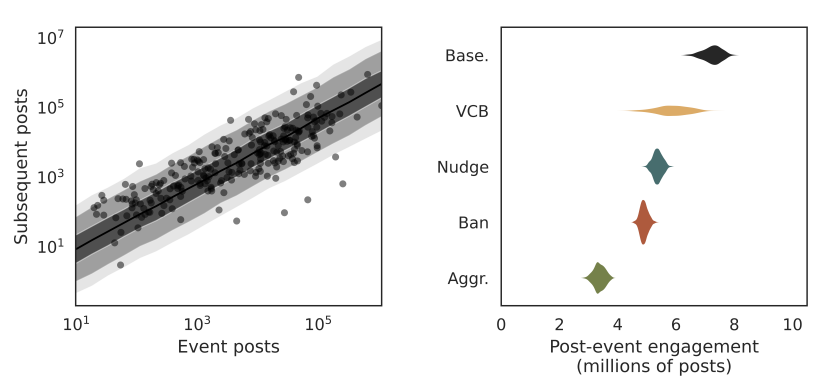

Figure 4. A) The impact of a modest combined approach to intervention (described in text, green) and each intervention applied individually $\mathbf{B}$ ) The impact of a more aggressive combined approach (described in text, green) and each intervention applied individually C) Relationship between engagement within the largest viral event for a given incident and subsequent engagement D) Expected post-event engagement given action taken during an event.

120 minutes. Among the content subjected to a viral circuit breaker, $20 \%$ is subsequently removed outright after four he further assume a 10\% reduction in individual more than $100 \mathrm{~K}$ followers. Our model reveals that even a modest combined approach can result in a $44.2 \%$ (89\% C.I. $[39,48.9])$ reduction in the total volume of misinformation igure 4A, Table S8).

We additionally consider a more aggressive version of a combined policy, applying viral circuit breakers to $10 \%$ of nt and reducing virality by $20 \%$ while cutting response threshold for the 3 -strikes policy to $50 \mathrm{~K}$ followers. This more aggressive approach dramatically reduced misinformation by $56.2 \%$ (89\% C.I. [51.3, 60.3], Figure 4B, Table S9). Similar efficacy from standalone approaches would be impossible ( $>40 \%$ Nudge) or require draconian removal of content and accounts.

One limitation of our model is that it relies on assumptions specific to periods of viral misinformation spread. In our dataset, only $36 \%$ of posts occur during the largest event for a given incident. Yet $25 \%$ of engagement occurs after the largest event. While our model cannot provide direct insight into how interventions will impact engagement during these periods, we can gain indirect insight by considering the relationship between the size of an event and subsequent discussion.

Our data demonstrate that the size of an event is strongly predictive of subsequent engagement (Fig. 4C, Bayesian LogNormal Regression $\beta=.94,89 \%$ C.I. [.92, .95], Table S10). Using this relationship, we can estimate subsequent discussion based on simulated, intervention-adjusted engagement during the largest event (See Methods). Through this, we reveal that the impact of interventions on post-event engagement is likely to be similar in magnitude to the efficacy during an event (Fig. 4D, S1).

\section{Conclusions}

Our derived model, grounded in data, provides quantitative insight into the relative efficacy of proposed interventions. We reveal that proposed interventions are unlikely to be effective if implemented individually at plausible levels through simulation. Effective removal of content or virality circuit breakers would require large teams, rapid turn-around times, and place content decisions squarely in the hands of private organizations. Nudges are promising but unlikely to be a panacea at known levels of efficacy ${ }^{5}$. Banning accounts appears the most workable solution but would require the removal of tens of thousands of users to be effective.

However, our results show that combining interventions at plausible levels of enforcement can effectively reduce the spread of viral misinformation. While it is unsurprising that multiple interventions outperform individual approaches, our findings provide insight into the magnitude of that difference. The efficacy of a combined approach depends not only on the nature of individual interventions but also on how they interact with one another, the dynamics of misinformation spread, event duration, user sharing behavior, user follower counts, and how these factors change throughout a disinformation campaign. In fitting our model to a large corpus of events during an active period of mis- and disinformation, our results are conditioned on much of this complexity. Further, by drawing from the empirical distribution of users' follower counts, our model indirectly and implicitly accounts for unseen behavioral patterns of users and changes to their follower count over time.

Our theoretical approach is limited in several key ways. Most notably, practical and ethical challenges preclude experimental validation of our theoretical findings. As our model evaluates the spread of viral misinformation at scale, experimental validation would require Twitter to adopt these interventions and apply them to millions of users. Moreover, limited transparency regarding interventions used by Twitter during the election raises the possibility that our model overestimates the efficacy of interventions that were cryptically in place ${ }^{12}$. Increased transparency on the part of platforms will be critical for future research on estimating the efficacy of interventions.

What further remains unclear is how changes in the magni- 
tude of events will impact longer-term dynamics of misinformation and translate to a reduction in harm. If implemented in tandem, multiple interventions may prove a sufficient shock to collapse the misinformation ecosystem altogether, as the shock-induced collapse is a central feature of complex systems ${ }^{16}$. For instance, subsequent events likely depend on the size of previous events and breaking that feedback could lead to greater than expected gains. However, this same body of literature suggests that an insufficient shock may yield only short-term changes as the system re-organizes and adapts.

We note that the results presented here rely on a simplified model of events on a single platform in a highly complex, multi-platform system. These types of simplifications are an inherent limitation of any approach-short of risky, largescale experimentation. However, abstract models of complex processes have proved essential to predicting the benefits of interventions on complex systems, from mitigating the spread of disease to stabilizing ecosystems ${ }^{17,18}$. Models provide particular utility when experiments are unethical and impractical, and costs of inaction are high. Given the substantial risks posed by misinformation in the near term, we urgently need a path forward that goes beyond trial and error or inaction. Our framework highlights one such approach that can be adopted in the near term without requiring large-scale censorship or major advances in cognitive psychology and machine learning.

\section{Methods}

\section{Data Collection and Processing}

Our dataset was collected in real-time during the 2020 US election. We relied on a set of 160 keywords to collect posts from Twitter's API (1.04B). Keywords were updated in response to new narratives, for instance, adding "sharpiegate" and related terms after false narratives emerged about the use of Sharpie markers invalidating ballots. Working with the Electoral Integrity Partnership, we cataloged instances of false or misleading narratives that were either detected by the team or reported by external partners ${ }^{2}$. This led to a large corpus of tickets associated with validated reports of misleading, viral information about election integrity.

Tickets that shared a common theme were consolidated into incidents. We developed search terms and a relevant date range for each incident to query posts from our tweet database. Incidents $(N=430)$ were generally characterized by one or more periods of intense activity followed by returning to a baseline state (Fig 1A). Search terms and descriptions of incidents are provided along with the data.

We then wished to extract segments of the time series that exhibit macroscopic features consistent with viral dynamics. More specifically, candidate events should exhibit quiescent periods before and after the event where our search terms return to baseline levels. However, multiple peaks may occur between these boundaries. To extract candidate events, we computed the raw time series of post volume per five minutes for each of our distinct incidents.
We identified events by finding the 5-minute interval within the aggregated time series with the largest number of collected posts. Other peaks in activity were considered part of separate events if they were at least $30 \%$ of the magnitude of the largest peak (to filter out noise). Event boundaries were determined as the points before and after the peak where the number of posts in 5 minutes was less than $5 \%$ of the maximum volume. If this did not occur within the range of data collection, the first (or last) time-point collected was used to denote the beginning (or end) of an event. Finally, events were required to last at least an hour (i.e., 12 data points). This process extracted 544 candidate events from 269 incidents.

\section{Statistical and Computational Model Model Derivation}

We then derived a model of spreading dynamics during viral misinformation cascades. We restricted our model to the dynamics of misinformation flow within a single event rather than longer timescale processes such as the adoption of beliefs and behaviors. The spread of beliefs and behaviors often require that multiple neighbors have adopted the state to spread (i.e., a complex contagion) ${ }^{19}$. Acceptance of a given misinformation narrative, for instance, can involve complicated cognitive processes involving partisan leanings, prior knowledge, attention, the message content, and a host of other factors 5,20

Re-sharing of information on Twitter, however, requires solely that a single neighbor has shared a piece of content for it to potentially be seen and retweeted ${ }^{21,22}$. Moreover, empirical work has demonstrated that out-degree (follower count) nearly linearly predicts engagement ${ }^{23,24}$. These features are hallmarks of simple contagions at the timescales of interest in our events. Following previous work, we, therefore, model the spread of viral misinformation as a simple contagion $^{11,25}$. At the core of our model is a latent virality parameter, $v$, which tracks the amount of attention a topic is garnering over time. Unlike typical compartment models, accounts vary widely in their out-degree from 0 followers to more than $100 \mathrm{M}$. In disease research, branching process models have incorporated various degree distributions to examine the role of super-spreaders ${ }^{17}$.

We built on models of super-spreading and leverage the fact that the out-degree of each account can be estimated by their total followers ${ }^{26,27}$. When a user posts during an event, our model assumes that virality is increased proportionately to their number of followers (i.e., the total exposed). However, network saturation and competition for attention with other topics can reduce virality over time. We incorporate this by adding a decay function, such that virality naturally decays over time. Together, growth from sharing and decay from saturation and competition define virality. Posts in a given timestep are predicted by virality in the previous timestep. These phenomena can be captured by a minimally parameterized branching process model, such that: 


$$
\begin{aligned}
\mathbb{E}\left[y_{t}\right] & =\exp \left(\alpha+\beta v_{t-1}\right) \\
v_{t} & =v_{t-1} \delta e^{-\lambda t}+x_{t} \\
x_{t} & =\log \left(\sum_{j=1}^{y_{t}} F_{j, t}\right)
\end{aligned}
$$

Where $y_{t}$ are the number of posts (i.e., retweets, tweets, replies, quote tweets) at 5-minute interval time $t, \alpha$ is the baseline rate of discussion, and $\beta$ is the effect of virality, $v$. Virality is a proxy for the total number of users at a given point in time exposed to and may propagate misinformation. Virality decays as an exponential function via $\delta$ and $\lambda$. Here, $\delta$ captures the baseline rate of decay per timestep, and $\lambda$ controls how that decay changes over the lifetime of an event. This could either be due to algorithmic processes favoring new content or user saturation for very large events. Every timestep, for each of $y_{t}$ accounts that posts, the log sum $\left(x_{t}\right)$ of their followers, $F_{j}$ is added to virality for the subsequent timestep.

We note that our model does not explicitly incorporate a network, as is common in many simulations of information and behavior spread online ${ }^{19}$. Our primary reason for doing this is that algorithmic filtering of content renders the true network topology unknown. Reconstructing a network would require additional epistemic assumptions, which could bias the results in opaque ways ${ }^{28}$. Moreover, research on disease has highlighted the utility of modeling interventions in the absence of network structure, notably when the degree distribution is known or approximated ${ }^{17}$.

Rather than solving this model analytically, we relied on a computational approach instead. We fit this model to each candidate event, including only those where the sampler performed well, and posterior predictive checks revealed adequate model fit. Of the 544 candidate events, our model performed well on 457 events $(\approx 9.7 \mathrm{M}$ posts $)$ of rapid misinformation spread. Modeling the spread of misinformation in this way allowed us to draw from an empirical distribution of follower counts specific to a given event and study the effect on engagement of banning users in a manner that is conditioned on their real-world patterns of behavior. This would not be possible with a simpler and perhaps analytically tractable model that approximates the degree distribution.

Our model was fit to each event using PyStan ${ }^{29,30}$. We fit events separately (rather than hierarchically) as they varied widely in their time scales, magnitudes, and context within the broader 2020 election cycle. These factors, combined with computational limitations, precluded a full hierarchical model from being feasible or appropriate. Similarly, our model was unlikely to be suitable for all events as it makes the assumption that post volume is well predicted by the number of previously exposed accounts on Twitter. If, for instance, an incident received substantial news coverage (i.e., Dominion software narratives) our model would likely fail.

To safeguard against this, we relied on a number of criteria to ensure model fit to a given event. Events were included in the final analysis if a) the posterior $89 \%$ C.I. of total posts contained the observed value and b) the chains successfully converged for all parameters $(\hat{R}<1.1)$ c) The fit did not contain divergent transitions and $d$ ) the event lasted longer than an hour (i.e., $>12$ data points to fit). Other than these criteria, events surrounding the dominion narrative were removed as they involved long periods high volume online discussion. This filtering process resulted in the inclusion of 457 events ( $84 \%$ of total events), and $\approx 9.7 M$ posts. We further verified that our model reliably generates observed engagement for all included events (Supplement Fig. S2).

\section{Statistical Model}

We derived parameters for our model statistically using a custom-written model $\operatorname{Stan}^{29}$. Posts $y_{i}$ at time $t$ are assumed to be distributed as a gamma-poisson mixture (i.e., negativebinomial) with expected value $\mu_{t}$ Specifically:

$$
\begin{aligned}
y_{t} & \sim \operatorname{NegativeBinomial} 2\left(\mu_{t}, \phi\right) \text { for } t=2 \ldots T \\
\mu_{i} & =\exp \left(\alpha+\beta v_{t-1}\right) \text { for } t=2 \ldots T \\
v_{t} & =v_{t-1} \delta e^{-\lambda t}+x_{t} \\
\alpha & \sim \operatorname{Normal}(-3,3) \\
\beta & \sim \operatorname{Normal}(0,3) \\
\delta & \sim \operatorname{Beta}(1,1) \\
\lambda & \sim \operatorname{HalfExponential}(1) \\
\phi & \sim \operatorname{HalfExponential}(1) \\
v_{1} & =x_{1} \\
x_{t} & =\log \left(\sum_{j=1}^{y_{t}} F_{j}+1\right)
\end{aligned}
$$

Where $\alpha$ is the baseline rate of detection for related keywords, and $\beta$ is the effect of virality, $v$ on posts in a subsequent timestep. Virality is calculated as a decaying function of $v_{t-1}$ and the log of the sum of account follower counts $F_{j}$ for posts in the previous timestep. One follower is added to each user to avoid an undefined value in timesteps with no followers. The $\log$ transform accounts for the link function (exp), transforming the linear model into an expected value for the Negative Binomial distribution. Given the wide range of possible event shapes, generic, weakly informative priors were chosen for all parameters. Models were fit using NUTS in Pystan with default sampling parameters ${ }^{29,30}$.

\section{Computational Model}

Our computational model relied on the posterior distributions of parameters obtained from fitting our statistical model separately to each event. For each simulation, one sample was drawn at random from the posterior for a given event. At $t=1$, the model was initialized with the volume of posts and total exposed users from the first timestep in which any posts were observed. At each subsequent timestep, our computational model predicted the number of new posts, $y_{t}$, by sampling from a negative binomial distribution as per our statistical 
model. For each of $y_{t}$ new posts, we drew a follower count from the actual distribution of accounts that retweeted for that event at that timestep. Doing so allowed us to control for the possibility that some accounts tend to appear earlier in a viral event. This process was repeated for the duration of the actual event.

We simulated removal of misinformation by simply setting $y_{t+1}=0$ after at a specified intervention time, $t$. Virality circuit breakers were enacted by multiplying virality at each timestep by a constant. For example, a $10 \%$ reduction in virality was implemented as $\hat{v}_{t}=v_{t}(1-.1)$. As with content removal, this occurred only after a specified timestep. In the case of the combined approach, virality circuit breakers (and subsequent removal) were employed at a given probability for each simulation run. We implemented nudges were through multiplying follower counts by a constant, reducing the pool of susceptible accounts (i.e., for account $j, \hat{F}_{j}=F_{j}(1-\eta)$ ). Finally, we implemented a 3 -strikes rule by identifying the third incident in which a given account appeared in our full dataset. They were removed from simulations for all events that occurred after their third strike.

Additionally, our model included a maximum value of twice the observed posts per time interval to account for a rare condition in which long-tail parameters would lead to runaway. This was observed to occur rarely enough to be challenging to quantify ( $<1 \%$ of model runs), but was implemented to reduce upward bias in control conditions. We did this to ensure conservative estimates of efficacy as interventions could reduce the possibility for runaway without meaningfully impacting engagement. Such a feature would be expected in any model of a growth process with pareto-like distributions of follower counts and spread at a given timestep (i.e., a negative binomial).

For the figures shown in the main text and the tables presented in the SI, we ran 500 simulations of all 457 events. For each run, we computed the cumulative engagement. The 500 simulations were summed across runs, from which we calculated the median and credible intervals. All simulations were done in Python.

\section{Model validation}

Some form of model validation strengthens any theoretical approach. As data-derived models of large-scale processes are uncommon in the social sciences, we offer some notes on validation and its limitations in this context. Ideally, our findings could be externally validated in an empirical setting. In our case, the gold standard would be to have Twitter implement our recommended policies in some locations but not others and examine subsequent engagement with viral misinformation.

Validation of this sort is both practically and ethically prohibitive. Ethically, the application of our theory to real-world social networks should occur after broader scientific scrutiny and not before publication. As these experiments impose actual costs on the individuals impacted by platform policies, complete evaluation by the scientific community is necessary to evaluate potential benefits and mitigate risks. Ethical challenges aside, such an experiment is impractical as it would require Twitter to rewrite its platform guidelines and hire fact-checkers at our suggestion. To the extent that Twitter conducts internal experiments, observational validation by the scientific community (i.e., natural experiments) is confounded by unseen changes in the user interface, algorithmic sorting, concurrent A/B testing, or other aspects of the experiment that are not disclosed to researchers.

Another possibility for external validation would be to conduct an online experiment in which a group of players is tasked with discerning truth in some domain. To mimic the behavior of our repeat offenders, a subset of players could be incentivized to spread misinformation. Experimental manipulations (e.g., removing players) would have the effect of reducing the amount of incorrect social information a player sees. A confirmatory finding in this context would simply provide evidence that, in this context, players respond to social information. Such an experimental approach would further lack the critical temporal and behavioral dynamics at the scale on which our model is conditioned.

A final approach to external validation could be an orthogonal dataset, for instance, COVID misinformation. However, the dataset presented in this paper is unique. It relied on collaboration between dozens of researchers and analysts at four institutions working over four months. In short, no such complementary dataset exists or can be compiled in a timely manner. Finally, our data were collected during a time when platform actions such as the removal of large accounts were rare. This is no longer the case, and a similar dataset would require additionally estimating the impact of ongoing removal. While this is likely possible, it would be an extension rather than a direct validation of our model.

This is a problem inherent to any data-derived model of a complex system at scale. Climate models suggest that reducing greenhouse gasses will slow climate change and highlight the relative efficacy of various approaches ${ }^{31}$. Yet empirical validation at scale would require convincing nations to experimentally reduce greenhouse gases alongside a control world where these policies are not applied. Similarly, an experiment involving altering conditions in an enclosed space may be consistent with data-derived models yet provide little additional insight $^{32}$. Further, there is no known orthogonal world in which models of anthropogenic disturbance can be externally validated. Nevertheless, models of greenhouse gas reduction remain our best hope at reversing climate change. Recent work has argued similar approaches are likely necessary for stewardship of our social systems ${ }^{13}$.

Here, we take a similar approach to climate models to validate our model internally (i.e., within our dataset). Climate models can be validated by allowing them to condition on data and then run freely for some time period. If the model successfully retrodicts conditions at a future point in time, it provides evidence that the model captures the dynamics of interest. We follow much the same approach here, simulating 
total engagement from the initial tweet throughout an event. This approach reveals that our model is quite capable of reproducing the observed patterns of engagement (SI Fig S2). We note that, unlike climate change, we can independently verify the model on 457 distinct events. Considering the relatively small number of parameters ( 5 in this model), this provides evidence that our model is capturing essential aspects of the underlying dynamics of the system as a whole.

\section{Post-event engagement}

Our model cannot directly evaluate post-event engagement, as it is designed to capture viral spreading dynamics rather than long, noisy periods of posting about a topic. These periods would be difficult to capture directly with a generative model, making it challenging to infer the impact of interventions on misinformation about a topic in general. However, there exists a quite regular relationship between the proportion of posts that occur within our definition of an event and those that occur subsequent to the event (Fig 4C).

We can leverage this fact to gain insight into how interventions may impact discussion following the viral periods we analyzed. To accomplish this, we used a Bayesian log-normal regression to estimate the effect of posts within the largest event on subsequent engagement (Table S10). We then use the posterior distribution from this model to estimate subsequent engagement as a function of engagement during our simulated events with intervention. This is summed across events to generate the estimates shown in in Figure 4D. This method provides insight, but we note it is limited by the assumption that the relationship between within- and post-event engagement is invariant to interventions. Further, it is limited by the extent to which our data collection process captured posts across the entire incident (i.e., event and subsequent posts).

\section{Code and Data Availability}

Code and data to reproduce the results are available on the Open Science Foundation (https://osf.io/2dcer/).

\section{Acknowledgements}

This work was made possible through the generous support from the John S. and James L. Knight Foundation, the UW Center for an Informed Public, the University of Washington eScience Institute, and Craig Newmark Philanthropies. We also thank our collaborators, the Stanford Internet Observatory, Graphika, DFRLab, and the Electoral Integrity Partnership. We further thank Carl Bergstrom, Iain Couzin, Fernando Rossine, Rachel Moran, and Kolina Koltai for their feedback.

\section{Author contributions statement}

J.B-C., A.B., and J.W. conceived of the study. J.S., A.B., M.W., I.K., E.S., and K.S., developed the dataset. J.B-C. and J.W. wrote the model and simulation code. J.B-C. drafted the initial manuscript and all authors were involved in subsequent revision.

\section{References}

1. Whitten-Woodring, J., Kleinberg, M. S., Thawnghmung, A. \& Thitsar, M. T. Poison If You Don't Know How to Use It: Facebook, Democracy, and Human Rights in Myanmar. The Int. J. Press. 25, 407-425, DOI: 10.1177/ 1940161220919666 (2020).

2. Election Integrity Partnership. The Long Fuse: Misinformation and the 2020 Election I Stanford Digital Repository. Tech. Rep., Center for an Informed Public, Digital Forensic Research Lab, Graphika, and Stanford Internet Observatory, Stanford Digital Repository (2021).

3. Koltai, K. VACCINE INFORMATION SEEKING AND SHARING: HOW PRIVATE FACEBOOK GROUPS CONTRIBUTED TO THE ANTI-VACCINE MOVEMENT ONLINE. AoIR Sel. Pap. Internet Res. DOI: 10.5210/spir.v2020i0.11252 (2020).

4. Pennycook, G. \& Rand, D. G. Fighting misinformation on social media using crowdsourced judgments of news source quality. Proc. Natl. Acad. Sci. United States Am. 116, 2521-2526, DOI: 10.1073/pnas.1806781116 (2019).

5. Pennycook, G. et al. Shifting attention to accuracy can reduce misinformation online. Nature 1-6, DOI: 10.1038/ s41586-021-03344-2 (2021).

6. Lazer, D. M. J. et al. The science of fake news. Sci. (New York, N.Y.) 359, 1094-1096, DOI: 10.1126/science. aao2998 (2018).

7. Velásquez, N. et al. Online hate network spreads malicious COVID-19 content outside the control of individual social media platforms. Sci. Reports 11, DOI: 10.1038/s41598-021-89467-y (2021).

8. Gollwitzer, A. et al. Partisan differences in physical distancing are linked to health outcomes during the COVID19 pandemic. Nat. Hum. Behav. 4, 1186-1197, DOI: 10.1038/s41562-020-00977-7 (2020).

9. Pennycook, G. \& Rand, D. G. Research note: Examining false beliefs about voter fraud in the wake of the 2020 Presidential Election. Harv. Kennedy Sch. Misinformation Rev. DOI: 10.37016/mr-2020-51 (2021).

10. Jin, F. et al. Epidemiological modeling of news and rumors on Twitter. In Proceedings of the 7th Workshop on Social Network Mining and Analysis, SNA-KDD 2013, 19, DOI: 10.1145/2501025.2501027 (Association for Computing Machinery, New York, New York, USA, 2013).

11. Wang, L. \& Wood, B. C. An epidemiological approach to model the viral propagation of memes. Appl. Math. Model. 35, 5442-5447, DOI: 10.1016/j.apm.2011.04.035 (2011).

12. Sanderson, Z., Brown, M. A., Bonneau, R., Nagler, J. \& Tucker, J. A. Twitter flagged Donald Trump's tweets with election misinformation: They continued to spread both on and off the platform. Harv. Kennedy Sch. Misinformation Rev. 2, DOI: 10.37016/MR-2020-77 (2021). 
13. Bak-Coleman, J. B. et al. Stewardship of global collective behavior. Proc. Natl. Acad. Sci. 118, e2025764118, DOI: 10.1073/pnas.2025764118 (2021).

14. Simpson, E. \& Connor, A. Fighting Coronavirus Misinformation and Disinformation - Center for American Progress. Tech. Rep., Center for American Progress (2020).

15. Bakshy, E., Mason, W. A., Hofman, J. M. \& Watts, D. J. Everyone's an influencer: Quantifying influence on twitter. In Proceedings of the 4th ACM International Conference on Web Search and Data Mining, WSDM 2011, 6574, DOI: 10.1145/1935826.1935845 (ACM Press, New York, New York, USA, 2011).

16. May, R. M., Levin, S. A. \& Sugihara, G. Complex systems: Ecology for bankers, DOI: 10.1038/451893a (2008).

17. Lloyd-Smith, J. O., Schreiber, S. J., Kopp, P. E. \& Getz, W. M. Superspreading and the effect of individual variation on disease emergence. Nature 438, 355-359, DOI: 10.1038/nature04153 (2005).

18. Levin, S. et al. Social-ecological systems as complex adaptive systems: Modeling and policy implications. Environ. Dev. Econ. 18, 111-132, DOI: 10.1017/ S1355770X12000460 (2013).

19. Centola, D. \& Macy, M. Complex Contagions and the Weakness of Long Ties. Am. J. Sociol. 113, 702-734 (2007).

20. Brady, W. J. et al. Emotion shapes the diffusion of moralized content in social networks. Proc. Natl. Acad. Sci. 114, 7313-7318, DOI: 10.1073/pnas.1618923114 (2017).

21. Bakshy, E., Rosenn, I., Marlow, C. \& Adamic, L. The role of social networks in information diffusion. In $W W W^{\prime} 12$ Proceedings of the 21st Annual Conference on World Wide Web, 519-528, DOI: 10.1145/2187836.2187907 (ACM Press, New York, New York, USA, 2012).

22. Kimura, M. \& Saito, K. Tractable models for information diffusion in social networks. In Lecture Notes in Computer Science (including subseries Lecture Notes in Artificial Intelligence and Lecture Notes in Bioinformatics), vol. 4213 LNAI, 259-271, DOI: 10.1007/11871637\{_\}27 (Springer Verlag, 2006).

23. Hofman, J. M., Sharma, A. \& Watts, D. J. Prediction and explanation in social systems. Sci. (New York, N.Y.) 355, 486-488, DOI: 10.1126/science.aal3856 (2017).

24. Lerman, K. Information Is Not a Virus, and Other Consequences of Human Cognitive Limits. Futur. Internet 2016, Vol. 8, Page 21 8, 21, DOI: 10.3390/FI8020021 (2016).

25. Juul, J. L. \& Ugander, J. Comparing information diffusion mechanisms by matching on cascade size. Proc. Natl. Acad. Sci. United States Am. 118, DOI: 10.1073/pnas. 2100786118 (2021).
26. Martin, T., Hofman, J. M., Sharma, A., Anderson, A. \& Watts, D. J. Exploring limits to prediction in complex social systems. In 25th International World Wide Web Conference, WWW 2016, 683-694, DOI: 10.1145/ 2872427.2883001 (International World Wide Web Conferences Steering Committee, Republic and Canton of Geneva, Switzerland, 2016).

27. Arif, A. et al. How information snowballs: Exploring the role of exposure in online rumor propagation. In Proceedings of the ACM Conference on Computer Supported Cooperative Work, CSCW, vol. 27, 466-477, DOI: 10.1145/2818048.2819964 (Association for Computing Machinery, 2016).

28. Butts, C. T. Revisiting the foundations of network analysis. Science 325, 414-416, DOI: 10.1126/SCIENCE. 1171022 (2009).

29. Carpenter, B. et al. Stan: A probabilistic programming language. J. Stat. Softw. 76, 1-32, DOI: 10.18637/jss. v076.i01 (2017).

30. Stan Development Team. PyStan: the Python interface to Stan (2018).

31. Saunois, M., Jackson, R. B., Bousquet, P., Poulter, B. \& Canadell, J. G. The growing role of methane in anthropogenic climate change, DOI: $10.1088 / 1748-9326 / 11 / 12 /$ 120207 (2016).

32. Foote, E. Circumstances affecting the Heat of the Sun's Rays. Am. J. Sci. Arts 22 (1856). 